Studies on Neutral Beam

Injection into the SSPX

Spheromak Plasma

R. Jayakumar, L.D. Pearlstein, T. A. Casper, T. K. Fowler, D. N. Hill, B. Hudson, H. McLean, J. Moller

October 19, 2007 
This document was prepared as an account of work sponsored by an agency of the United States government. Neither the United States government nor Lawrence Livermore National Security, LLC, nor any of their employees makes any warranty, expressed or implied, or assumes any legal liability or responsibility for the accuracy, completeness, or usefulness of any information, apparatus, product, or process disclosed, or represents that its use would not infringe privately owned rights. Reference herein to any specific commercial product, process, or service by trade name, trademark, manufacturer, or otherwise does not necessarily constitute or imply its endorsement, recommendation, or favoring by the United States government or Lawrence Livermore National Security, LLC. The views and opinions of authors expressed herein do not necessarily state or reflect those of the United States government or Lawrence Livermore National Security, LLC, and shall not be used for advertising or product endorsement purposes.

This work performed under the auspices of the U.S. Department of Energy by Lawrence Livermore National Laboratory under Contract DE-AC52-07NA27344. 
Studies on Neutral Beam Injection into the SSPX Spheromak Plasma

R. Jayakumar, L.D. Pearlstein, T.A. Casper, T.K. Fowler, D.N. Hill, B. Hudson, H. McLean, J. Moller

Lawrence Livermore National Lab, Livermore, CA

\begin{abstract}
:
In the Sustained Spheromak Physics Experiment, (SSPX) [“Improved operation of the SSPX spheromak ",R.D. Wood, D.N. Hill, E.B. Hooper, S. Woodruff1, H.S. McLean and B.W. Stallard, Nucl. Fusion 45 1582-1588 (2005)], plasmas with core electron temperatures reaching up to $500 \mathrm{eV}$ at densities of $10^{20} / \mathrm{m}^{3}$ have been sustained for several milliseconds, making them suitable as targets for neutral beam injection. High performance and further progress in understanding Spheromak plasma physics are expected if neutral beams are injected into the plasma. This paper presents the results of numerical $1.5 \mathrm{D}$ modeling of the plasma to calculate neutral beam current drive and ion and electron heating. The results are presented for varying initial conditions of density, temperatures and profiles and beam energy, injection angle and power. Current drive efficiency (Ampere/Watt of absorbed power) of up to 0.08 can be achieved with best performance SSPX shots as target. Analyses of neutral beam heating indicate that ion temperatures of up to $1.5 \mathrm{keV}$ and electron temperatures of up to $750 \mathrm{eV}$ can be obtained with injection of about $1 \mathrm{MW}$ of neutral beam for 5-10 ms and with diffusivities typically observed in SSPX. Injection targeting near the magnetic axis appears to be the best for heating and current drive. Effect of the current drive and evolution of SSPX equilibrium are discussed.
\end{abstract}

\title{
I Introduction:
}

The spheromak is a compact self-organized toroidal plasma configuration in which confining magnetic fields are produced by poloidal and toroidal currents flowing in the plasma [1] with no toroidal magnetic field coils linking the plasma and no central solenoid driving a toroidal current providing a compact geometry with inexpensive coil set. A spheromak is formed and sustained by injecting magnetic helicity $(K=V \boldsymbol{A} \cdot \boldsymbol{B} \mathrm{d} V)$ from a magnetized coaxial plasma gun into a conducting confinement region (flux conserver). Through magnetic fluctuations and associated reconnection the injected plasma then relaxes into an axisymmetric toroidal geometry. The equilibrium profiles in the spheromak are nearly force free-a so-called Taylor relaxed state [2]. The sustained spheromak physics experiment (SSPX) [3] uses a plasma gun and sets of coils to form a spheromak and has been used to study the formation, sustainment and confinement studies. Confinement improvements in SSPX give peak $\mathrm{Te} \sim 500 \mathrm{eV}$ and core electron thermal diffusivities of $\chi_{\mathrm{e}}<1-10 \mathrm{~m}^{2} / \mathrm{s}$. However, SSPX temperature data are bounded by $\beta_{\mathrm{e}} \sim 5 \%$ since the increase in temperature is obtained through increasing the magnetic field strength $\left(T \sim B^{2}\right)$. Since the generated magnetic field and the plasma current are in proportion and since the ohmic power is proportional to the current, $\mathrm{T} \sim \mathrm{I}_{\mathrm{p}}{ }^{2}$, which is similar to an ohmic 
confinement scaling[4]. Therefore, present experiments are unable to determine if the plasma temperature is limited by stability or due to ohmic confinement scaling. An auxiliary heating source where the power is uncoupled to the plasma current is needed to discriminate between these two limitations. With an auxiliary heating source, the characteristics of an L-mode regime for spheromak could be studied and compared with other devices. At present, the Spheromak sustainment is constrained by the gun and modification of the plasma characteristics and profiles independently of the formation and the gun is not possible. Therefore, an auxiliary current drive source is needed for adding parallel current and altering the equilibrium profile to study the dynamics of helicity injection and/or current and equilibrium evolution. Such a current drive may also allow access to different spheromak equilibria and an ability to make moderate changes to the control may also provide some ability to control the evolution of safety factor and onset of MHD instabilities. If a significant current drive can be achieved, it can replace helicity injection, which potentially opens field lines, for spheromak sustainment

Injection of neutral beam over several milliseconds would provide such a plasma heating and current drive source. In addition, the source enable measurement of ion temperature and observation of coherent and turbulent flucuations and determination of current profile using Motional Stark Effect diagnostic techniques. The paper presents the results of numerical calculations using the code CORSICA [5]. Section II describes the conditions for the calculations and code assumptions. Section III describes the results of the calculation for current drive, Section IV describes the results for neutral beam heating and current drive and Section $\mathrm{V}$ provides conclusions and discussions.

\section{SSPX device and conditions for NBI calculations:}

An overview of the SSPX device and operation is given in ref $[3,6]$. The SSPX device produces $1.5-8.0 \mathrm{~ms}, 1 \mathrm{~m}$ diameter by $0.5 \mathrm{~m}$ high spheromak plasmas with a plasma minor radius of $0.23 \mathrm{~m}$ and a major radius of $0.31 \mathrm{~m}$. Figure 1 shows a cross section of the SSPX identifying the major hardware components along with a typical MHD equilibrium In SSPX, an outer electrode of the coaxial region is contiguous and electrically connected to the outer flux conserver. The plasmafacing surfaces of the copper flux conserver are tungsten-coated ( $100 \mu \mathrm{m}$ thick) to reduce sputtering. A adjustable vacuum 'bias' flux configuration is provided. a spheromak plasma is formed when gas is injected into the coaxial region and a voltage applied to the inner electrode (the discharge cathode). The resulting plasma is rapidly ejected into the flux conserver when the current rises above the ejection threshold after $\sim 150 \mu \mathrm{s}$. The ejection threshold current depends on the strength and configuration of the vacuum magnetic field. The spheromak plasma decays resistively with a time constant of several milliseconds, and since the electron temperature ( $\mathrm{Te}$ ) is lower at the edge than at the magnetic axis, the spheromak current profile gradually becomes more peaked leading to a collapse due to a global instability The onset of this instability is avoided by using a sustainment capacitor bank to maintain an edge current, which provides a pulse 
of upto nearly $5 \mathrm{~ms}$. Additional modular banks have recently been added to maintain a spheromak for nearly $8 \mathrm{~ms}$.

The toroidal current and internal magnetic field profiles of the resulting spheromak plasma are inferred from edge poloidal magnetic measurements by 20 toroidally and poloidally distributed magnetic probes. A 2D MHD equilibrium is reconstructed using the CORSICA code, assuming that $\lambda=\mu_{0} J / B$ is a flux function of the form $\lambda=\lambda_{\text {edge }}\left(1+\alpha \psi^{n}\right) /(1+\alpha)$, where $\lambda_{\text {edge }}=\lambda_{\text {gun }}=I_{\text {gun }} / \psi_{\text {gun }}$, where $I_{\text {gun }}$ and $\psi_{\text {gun, }}$ are the gun current and gun poloidal flux and $s$ is the poloidal flux function.. The equilibrium reconstruction includes plasma current on the open field lines as shown in figure 1. The electron temperature and density are measured using Thomson scattering technique. The electron density is closely related to formation conditions, wall conditioning and presence of impurities. High electron temperatures (low diffusivities) are obtained at low to moderate densities when other conditions are optimized. Figure 2 shows the fitted electron temperature and density profile at the Thomson laser pulse time, the electron pressure and q (safety factor) profiles for two discharges 14590 (plasma current on closed field lines $=370 \mathrm{kA}$ ) and 20217 (plasma current $=280 \mathrm{kA}$ ). At present, there is no ion temperature diagnostic in the machine, but indications are that the temperature is not lower than half of the electron temperature. In computing these equilibria, the ion and electron temperatures are assumed to be equal. (The pressure has only a small effect on the equilibrium q profile due to the dominance of the current profiles and the equilibrium is force free to the zeroth order).

Neutral Beam Injection and plasma response:

The injection of a beam of fast neutral particles results in the ionization and a confined population of fast-ions. Some of the circulating fast-ion current is cancelled by the elctron response which depends on the ratio of beam charge to the Zeff of the discharge. Methods for obtaining the neutral beam current drive have been described [7-9] with solution to the Fokker- Planck equations and good approximations to resulting integrals. Calculations of neutral beam injection in Spheromaks have also been reported [10], for a theoretical equilibrium. In the present calculations, the beam package built into the CORSICA code was used. The package uses the Monte-Carlo code NFREYA [11] to obtain the slow down distribution of fast-ions. A new fast-ion orbit-following algorithm was recently added to CORSICA to account for the low field, low aspect-ratio magnetic topology of the spheromak configuration, in which the particle orbits can be quite exotic compared to a tokamak.[12] In this new model The neutral from the CX deposition is assumed to be ionized in place, a highdensity approximation. The fast ions are modeled to occupy orbit with constant canonical angular momentum at the birth time or at a chosen location. The fast ions are assumed to have a dragged down distribution. Rates for momentum and energy transfer to plasma electron and ions are calculated from this distribution function which then allows the fast-ion population and driven 
current to reach steady states over a relaxation time. (When the beam is turned off, the same rates are operative and the fast-ion population and the current decay at this rate). The particle weights in each flux zone (the sampling bin) are proportional to the time spent in the zone. From this information the fluxsurface-averaged profiles are obtained and fed into the appropriate transport equation.

The CORSICA code can be used in a single time slice mode to calculate the neutral beam current drive and input power. But, CORSICA transport calculation in which the current, density and temperature evolution simultaneously and reconstruct equilibria for each time step advanced, gives a self-consistency . This also allows the determination of various relaxation times and effect of short beam pulse(s). Carrying out this analysis for a model Spheromak case poses some difficulties; (1) The density transport is poorly understood for most plasmas because of the poor identification of sources. This is particularly true of small devices such as the SSPX. Hence, in the present calculations, the electron density and impurity densities and their profiles are held constant. In a typical experiment with a moderate pulse length, a moderate control is likely to be achieved by wall conditioning, gas puff control. For actual experiments with NBI , the NBI calculations would be carried out with measured profiles as inputs. (2) Spheromak poses a particular difficulty in evolving equilibria. The applied loop voltage in is expected to be zero in SSPX and therefore there is no external parallel current drive. In such a situation, a nominal Ohm's law dictates that poloidal flux would diffuse to the core and the safety factor would drop rapidly, as opposed to the observed slow evolution of the plasma equilibrium. The $\mathrm{q}$ profile evolution of the discharge 14590 (see below) using neoclassical Ohm's law is shown in Fig.3. This shows that within few hundred microseconds the minimum safety factor would drop by nearly 0.25 and current distribution would peak in the core. This is contrary to the experiment, where the safety factor changes at nearly one tenth of his rate. The additional terms that cause the Spheromak sustainment (a self consistent helicity drive) have not been identified and the profile of the EMF due to the drive has not been determined experimentally. (It is speculated that Spheromaks like RFPs are perhaps sustained by a dynamo action or helicity injection arising out of the large poloidal current from the gun). Further, it is unknown how the Spheromak equilibrium would evolve in the presence of NBI. Therefore, except for one case study, the equilibrium safety factor is held constant is held constant while the current drive and electron and ion heating are calculated. This allows pressure to change in presence of heating and the evolution is accompanied by small changes in current profile in the recalculated equilibrium (using the built-in TEQ code). Again, a more consistent calculation would be carried out if and when sufficient information is available to include or model the safety factor evolution.

Fixing of electron density and impurity density profiles leads to the fixing of the (hydrogen) ion density profile. This, in turn, means that as fast-ions 
accumulate the code throws out corresponding number of plasma hydrogen ions. This hollows out the plasma hydrogen ion profiles since the fast-ions accumulate mainly in the core. In the code, the fast-ions are included as target ions for the injection and the neutral beam injection and slow-down remain unaffected. For high powers, the inventory of plasma hydrogen ions being replaced can run out and once the local hydrogen density at any radius drops to near zero, the fast-ion density may be assumed to saturate and any additional fast-ion inventory can be accounted for by broadening the fast-ion density. However, in these calculations the NB power and density are chosen such that the fast-ion density does not have to saturate anywhere. Allowing the fast-ion density profile to broaden might give unphysical results. The alternative of allowing the total densities to increase poses a different difficulty, namely that the pressure increases too much and TEQ reconstructions with fixed safety factor profile can fail. The fixing of the electron and impurity profile and equilibrium safety factor represents a reasonable choice for calculating the current drive and heating for a specified condition that corresponds to the plasma state at the final time step.

The calculations reported assume that the beam is injected at the midplane of the Spheromak and the impact parameter (radius of tangency to the toroidal direction ) is given by $0.65^{\star} \sin (\theta)$ where $\theta$ is the injection angle. The beam is assumed to be $0.1 \mathrm{~m}$ in diameter and hydrogen species is used. Different angles of injections and energies are examined. Calculations were carried out for the two discharges shown in Fig. 2. For the case of 14590, calculations were also carried out with $70 \%, 100 \%$ and $150 \%$ of the density and temperature. In addition, a flat density profile given by $\left(1-0.9 \psi^{5}\right)$ with an average density of $4.5 \times 10^{19} / \mathrm{m}^{3}$ (same as that of the profile in Fig. 2 for discharge 14590), was also investigated to see profile effects.

One of the most important parameters in calculating the temperature rise and change in the temperature profile is the electron and ion diffusivities. For SSPX, the electron (effective) diffusivity is obtained from energy balance between thermal diffusion and joule heating in the closed flux region. A range of results for the resulting diffusivities is given in ref.[7]. The computed diffusivity profile for the two discharges shown in Fig. 2 are shown in Fig. 4. Since the ion temperature is not measured for these discharges, calculations are done aiming for $50 \%$ and $100 \%$ of the electron temperature. Neutral beam current drive calculations are carried out by evolving the temperatures using a diffusivity profile

\section{Results of Calculation of the Neutral Beam Current Drive:}

In this section we report the results of calculations of neutral beam current drive while holding the equilibrium safety factor, density and temperature profiles constant as initially specified. This permits the study of the effect of the density and temperature on current drive. 
Fig. 5 shows the variety of orbits for the spheromak. Fig. 6 and 7 show the results of the calculations for the equilibrium for 14590 (shown in Fig. 2) and for a neutral beam power of only $100 \mathrm{~kW}$ so that there is only a small reduction in plasma ion densities. Fig. 6a shows the current driven (amps) for one watt of power injected (solid) and one watt of power absorbed (dashed) vs injection angle. The maximum efficiency of drive is obtained for an injection angle of about 35-37 degrees which correspond approximately to injection through the magnetic axis. This result is same for both peaked (solid) density profile shown in Fig. 3 and for an analytical flat density (dashed) density profile. The flat density profile has a higher efficiency of current drive. Fig. $6 \mathrm{~b}$ shows the weak dependence of the absorbed power on the injection angle, except for large angles where the shine through (reduction in plasma thickness) becomes large.

Fig. 7a shows the current drive efficiency per watt of power absorbed for 3 temperatures and 3 densities (nominal, 33\% less than nominal and 50\% more than nominal shown in Fig. 2 for discharge 14590 and peaked density profile). As expected, the efficiency increases with increasing temperature and decreases with increasing density. Zero D calculations would predict an NBCD efficiency proportional to $\mathrm{T} / \mathrm{n}$ ( $\mathrm{T}$ being the temperature and $\mathrm{n}$, the plasma density) (Ref) and as shown in Fig $7 \mathrm{~b}$ this is approximately true. However, the results with lower efficiency for the same $\mathrm{T} / \mathrm{n}$ correspond to higher density and therefore there is a stronger than inverse dependence on density. These plots roughly correspond to a $\mathrm{I}_{\mathrm{NB}} / \mathrm{P}_{\mathrm{abs}} \sim \mathrm{T}^{0.98} / \mathrm{n}^{1.2}$.

Fig. 8 shows the profile of the neutral beam current density for different angles at $25 \mathrm{keV}$ and $37.5 \mathrm{deg}$ injection and the peaked density profile. The current density profile is essentially near the axis for all cases and for off-axis injection angles, there is a strong reduction of current drive close to the axis and no appreciable increase in off-axis current drive. Very similar current density profiles, however with larger current drive efficiency at the optimum angle, are obtained for the flat density profile.

Fig. 9 shows the dependence of NBCD efficiency on the beam energy. Above $30 \mathrm{keV}$, the dependence of the current per watt absorbed remains nearly constant, while current drive per watt of injected power is maximum at about 40 $\mathrm{keV}$. As expected, as the energy is increased, the absorption is reduced and the profile of the current density widens slightly as the energy is increased.

The above results were for an injection power of $100 \mathrm{~kW}$ where the change in the core plasma ion density is limited to a few percent. However, as the power is raised the fast-ion density becomes comparable to plasma ion density. Fig.10 shows the plot of fast ion density profile for different injected power, for the case of $37 \mathrm{deg}$ and electron density of 33\% less than the nominal and electron temperature of 50\% higher than nominal, for discharge 14590 . Since the total ion density is fixed, the thermal ion density is reduced at each step by the amount of fast-ion density and once the plasma ion density reaches a 
minimum preset value at a radius, the fast-ion density is not allowed by the code to increase at that radius and further increases in the fast-ion would only widen the fast-ion profile and cause reduction in current drive and heating (for example the case of $1.35 \mathrm{MW}$ ). The above situation is driven by code settings and therefore variations of the above could be modeled. There is another setting that affects the efficiency of the current drive, namely that of including or not including the fast-ion pressure in calculating the equilibrium. Obviously, if the fast-ions are treated as having thermal ion temperature then the equilibrium does not change and the dependence of current drive on beam power remains linear. However, if the fast-ion pressure is included as such while maintaining the total plasma ion density, the equilibrium core pressure increases leading to a changed equilibrium current density (while safety factor profile is held fixed by the TEQ) with a reduced current density in the core there is a non linear increase of current drive efficiency with increasing beam power. Fig. 11 shows the profiles of the total initial pressure and pressure vs the toroidal flux coordinate (square root of Toroidal Flux), with NBI of $900 \mathrm{~kW}$ with $25 \mathrm{keV}$ energy at an injection angle of $37 \mathrm{deg}$ for the nominal profiles of discharge 14590. It is clear that the fast-ion pressure dominates the plasma pressure. Fig, 11b shows the resulting change in equilibrium total parallel current density profile. It is evident that several hundred kilowatts of neutral beam injection would be extremely perturbative even without including self organizing effects added and many effects would be observed in conjunction. For the case, when the equilibrium is recalculated including fast-ion pressure and no change in target density profiles, the current drive efficiency increases with injected power and this is shown in Fig. 12.

\section{Neutral Beam Heating and Current Drive:}

In the following section, results of CORSICA transport calculations to obtain electron and ion heating and the accompanying current drive consistent with heating. Again, since CORSICA transport does not incorporate the physics of Spheromak equilibrium evolution, the equilibrium safety factor is held fixed while the time steps are taken. Since the heating would depend on injected power calculations are made for 2 values of power $(450 \mathrm{~kW}$ and $900 \mathrm{~kW})$. There would again be some difference depending upon whether fast-ion pressure is included or not. Since the temperature evolution would depend on the thermal diffusivities these are also varied to check the effect of diffusivity profiles. Calculations are also made for the discharge 20217 for comparison with that for discharge 14590.

The procedure for the heating and current drive calculations are as follows: The initial conditions correspond to Figs. 3. The diffusivities shown in Fig. 4 are scaled to $1.0,0.4$ with ion and electron diffusivity profiles held the same. The initial estimated diffusivity profiles are obtained from energy balance between thermal conduction and Joule heating. Since no ion effects are included in this estimate while CORSICA transport calculations do and also because 
transport calculations are sensitive to temperature profiles, the initial diffusivity profile is not necessarily consistent with CORSICA transport. Therefore, a number of time steps for a given scaling of the diffusivity profile to let the temperature profiles settle down and then the neutral beam injection is started and the heating and current drive corresponding to the steady state electron and ion temperature profiles, is obtained. The peak temperatures and profiles at the start of injection are therefore different for each scaled diffusivity. It must be noted, there are small changes (a few \%) in total current density profile because of the neutral beam current drive and rise of bootstrap current, while the safety factor profile and pressure profile are held constant.

Fig.13 shows the history of the central electron (solid) and ion temperatures (dashed) is shown for four cases: $450 \mathrm{~kW}$ and $900 \mathrm{~kW}$ of NBI power and for diffusivity scales of 1.0 and 0.4 for profiles of 14590 . The initial phase before injection shown in arrow, the time corresponds to iterations to obtain a steady state profile. As can be seen, with an initial central temperature of about $360 \mathrm{eV}$ similar as 14590, an ion temperature of close to $1 \mathrm{keV}$ and an electron temperature of $750 \mathrm{eV}$ can be achieved after $5 \mathrm{~ms}$ of $900 \mathrm{~kW}$ beam injection. For this case, about $8 \%(25 \mathrm{kA})$ of the plasma current is driven by the beam and $100 \%$ of axial current density would be beam driven. These numbers are somewhat higher when fast-ion pressure is included in reconstructing the equilibrium.

Fig. 14 shows the initial (no symbol) and final (9 millisecond injection) (circle) electron (solid) and ion (dashed) temperature profiles for each of these cases. The injection essentially heats the core of the plasma. Fig. 15 shows the profile of the total and driven parallel current densities. For the case of diffusivity scaled by a factor 0.4 and injection of $900 \mathrm{~kW}$, the core driven current density is equal to the total current density. If achieved, this would present an interesting regime.

A strong heating and current drive was obtained for the discharge 20217 with electron temperature close to achieved in the experiment ( $460 \mathrm{eV}$ peak) and diffusivity scaling factor of 0.4 . With only $450 \mathrm{~kW}$, the on-axis NB driven current density is about $75 \%$ of the initial total current density and a current drive efficiency $\left(\mathrm{I}_{\mathrm{NB}} / \mathrm{P}_{\mathrm{abs}}\right)$ of 0.074 with $22 \mathrm{kA}$ of driven current in a $320 \mathrm{kA}$ discharge. An ion temperature of nearly $1.5 \mathrm{keV}$ was reached in $9 \mathrm{~ms}$. These results are shown in Fig. 16.

Another interesting result is shown in Figure 17 corresponding to an injection of $1.8 \mathrm{MW}$ at 45 degrees. (This high power calculation was possible, since the NB efficiency is poorer and the fast-ion density remained well below the initial thermal ion density). When compared to the 37 deg injection, the temperature profiles are also broad and a hollow and broad driven current density of nearly $25 \%$ is obtained (see Fig. 6 )

Results of calculation for an injection of a short $1.2 \mathrm{~ms}$ long pulse of 600 
$\mathrm{kW}$ at $22 \mathrm{keV}$, (corresponding to a beam that may be readily available) are shown in Fig. 18. This shows that the effect of injection would be to raise temperatures significantly and a small current would also be driven. For the assumed diffusivity, it is clear that the effect of the heating persists for a few milliseconds.

\section{Discussions:}

The foregoing results show that a neutral beam injection of moderate voltage and power will add substantial heating to the plasma if the conditions of self organization in the SSPX Spheromak continue to be sustained in presence of NBI. As shown, even a short pulse may bring the Spheromak conditions into new regimes of temperature and beta. In general, the introduction of a core current drive should alter the induced EMF profiles and new regimes of current evolution may be obtained. In the above calculations, the safety factor and total plasma current of the fixed-boundary equilibrium were held fixed and except for one study, the pressure profile was also fixed, since the physics of flux evolution is not modeled in the neoclassical resistive flux diffusion in Corsica. However, the Speromak equilibrium in presence of NBI will most likely be different from the initial equilibrium. Therefore, these calculations correspond to the case where the final state represents the equilibrium (initial which is same as final) in presence of NBI.

However, instead of time evolving the equilibrium, the equilibrium can be recalculated with the fast-ion pressure and added current. Such an equilibrium reconstruction is presented for the nominal case of unscaled density and diffusivity for an injected power of $450 \mathrm{~kW}$, is shown in Fig. 19. It is clear that even with a modest injection of current, the safety factor profile changes significantly. The core change in safety factor is actually held lower by the increase in core pressure due to fast-ion which broadens the current profile.

Other methods such as hyper-resistivity models may be used to simulate current evolution in presence of NBI. An example [14] is to write the Ohm's law as

$$
V_{L}=\frac{\partial \Psi}{\partial t}=2 \pi\left[\eta \frac{<J . B>}{<B . \nabla \varphi>}-\frac{\partial}{\partial \Phi}\left(\mathrm{H}<|\nabla \Phi|^{2}>\frac{\partial V}{\partial \Phi} \frac{\partial \lambda}{\partial \Phi}\right)\right]
$$

where $V_{\mathrm{L}}$ is the loop voltage at a given surface, $\Psi$ and $\Phi$ are the poloidal flux at that surface, $\mathrm{J}$ and $\mathrm{B}$ are local current density and magnetic fields, $\mathrm{V}$ is the flux surface volume and $\lambda$ is the Spheromak equilibrium parameter. $H$ is the hyper resistivity parameter whose profile is undetermined and the determination of which could correspond to a model of Spheromak decay. (As stated above, with the hyper-resistivity term set to zero, the core safety factor would continuously drop at a much faster rate compared to observed rate, since the Spheromak sustainment with edge gun current is not (cannot be) modeled in CORSICA.) CORSICA thermal and current transport calculations with hyper-resistivity 
terms are expected to be possible in the future as the code capability is improved.

The thermal diffusivities can be modeled with Gyro Landau Fluid or gyrokinetic codes. These efforts are also planned for the future. Additional effects in hyper-resistive models may also be required to conform to Spheromak conditions. It may be noted that in the above calculations the ion and electron diffusivities were taken to be equal and usually the ion diffusivities are smaller than that for electrons. With neutral beam injection, the ratio of ion to electron temperature increases in the core and this will lead to further decrease in diffusivities particularly for the ions and may even lead to transport barriers. In the region of reversed safety factor such reduction in transport would be favorable [15].

\section{Conclusions:}

Neutral beam current drive and heating for the SSPX Spheromak have been investigated using the CORSICA transport code for different scaled values and profiles of densities and temperatures. There is a somewhat stronger than proportional increase in current drive efficiency with decreasing density. With a sustained injection of $450 \mathrm{~kW}$, electron temperatures in excess of $750 \mathrm{eV}$ and ion temperature of about $1.5 \mathrm{keV}$ was obtained using a high performance SSPX discharge as target and about $0.074 \mathrm{~A} /$ Watt of absorbed power. It is shown that even a short pulse of about $1 \mathrm{~ms}$ with $600 \mathrm{~kW}$ power raised the temperatures by over $100 \mathrm{eV}$.

\section{References}

1. E. B. Hooper, L. D. Pearlstein and R. H. Bulmer, MHD equilibria in a spheromak sustained by coaxial helicity injection, Nucl. Fusion 39 (1999) 863.

2. T.R. Jarboe, Plasma Physi. Controlled Fusion 36, 945(1994)

3. R.D. Wood, D.N. Hill, E.B. Hooper, S. Woodruff1, H.S. McLean and B.W. Stallard, Nucl. Fusion 45 1582-1588 (2005)

4. R.J. Goldston, Plasma Phys. Controlled Fusion 26,87 (1984)

5. James A. Crotinger, et al., CORSICA: A Comprehensive Simulation of Toroidal Magnetic-Fusion Devices, Report UCRL-ID-126284, Lawrence Livermore National Laboratory, CA, April, 1997.

6. H.S. McLean,S. Woodruff, E.B. Hooper et al., Phys.Rev.Lett 88, 125004 (2002)

7. D.F.H. Start and J.G. Cordey, Phys. Fluids 23,1477(1980)

8. D.R. Mikkelsen and C.E. Singer, Nuclear Tech. Fusion 4, 237(1983)

9. P.A. Politzer and G.D. Porter, Nuclear Fusion 30,1605(1990)

10. A F Lifschitz, R Farengo, and N R Arista1, Plasma Phys. Control. Fusion 44,1979 (2002) 
11, Goldston, R.J., et al., J. Comp. Physics 43, 61(1981)

12. L.D. Pearlstein, 33d EPS Conference on Plasma Phys. Rome, 19 - 23 June 2006 ECA Vol.30I, P-5.128 (2006).

13. J.D, Cllen, J.D., et al., IAEA-Cn-33A16-3, (1974)],

14. E.B. Hooper and L.D. Pearlstein, Plasma Phys. Reports 38765(2002)

15. R. Jayakumar, Fusion Sci. Tech, 46 ,225(2004) 


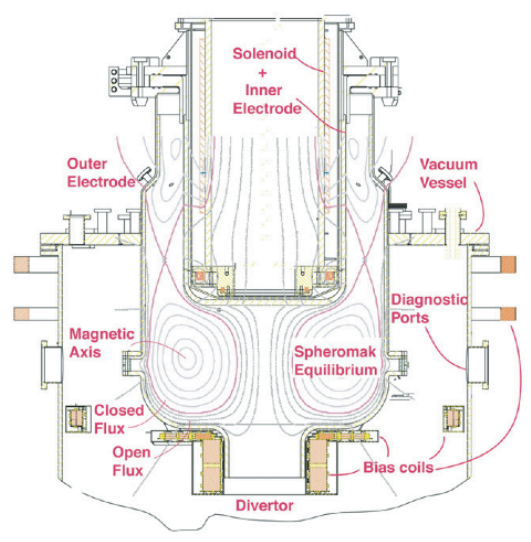

Fig. 1 Schematic of the SSPX Spheromak with example equilibrium
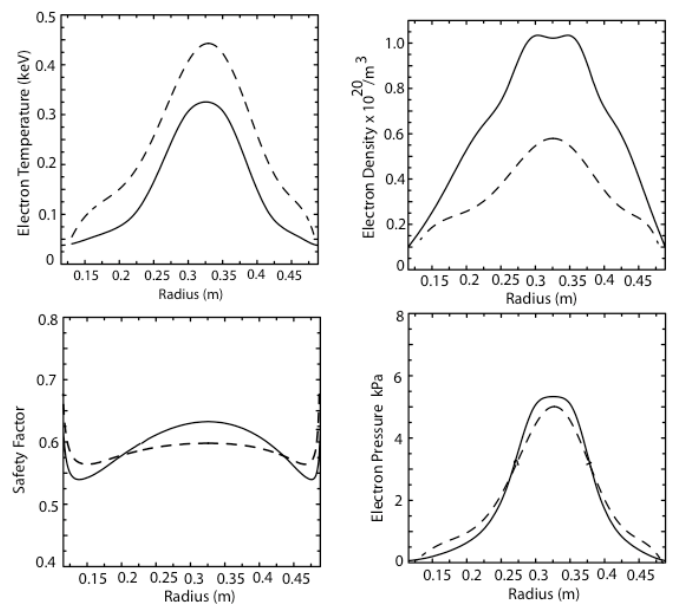

Fig. 2 Profiles of electron temperature, density, safety factor and electron pressure for two discharges 14590 (solid) and 20217 (dashed) 


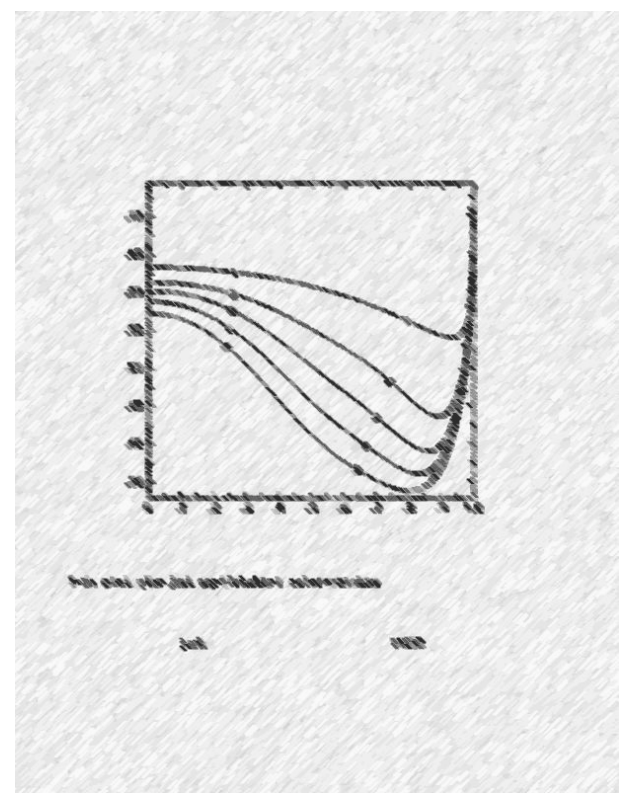

Fig. 3. Safety factor vs toroidal flux coordinate (square root of toroidal flux) every 100 microsec due to flux transport in CORSICA

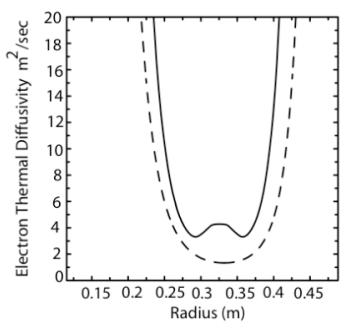

Fig.4 Electron diffusivity profile for 14590 (solid) 20217 (dashed) with energy balance 

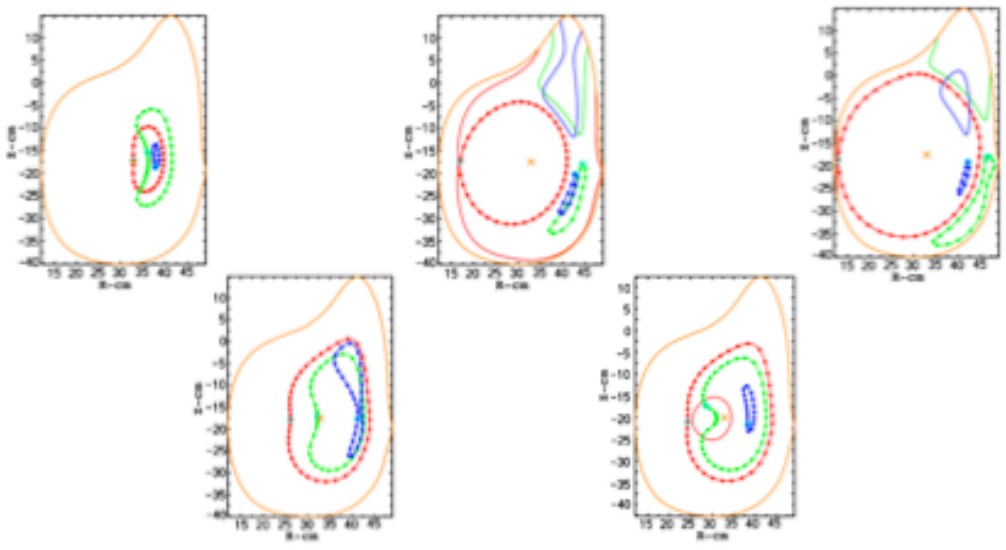

Fig. 5 Examples of fast-ion orbits for different birth locations 

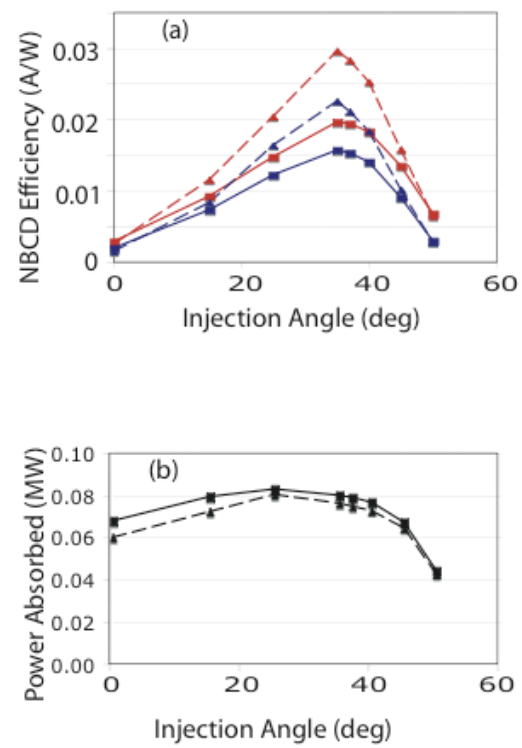

Fig.6 Top - NB Current drive efficiency vs injection angle ,Amps/Watt of injected power (blue), Amps/Watt of absorbed power (red) for 14590 density and temperature profiles (solid), for flat density and 14590 electron temperature profiles (dashed); Bottom power absorbed for $100 \mathrm{~kW}$ of injection.

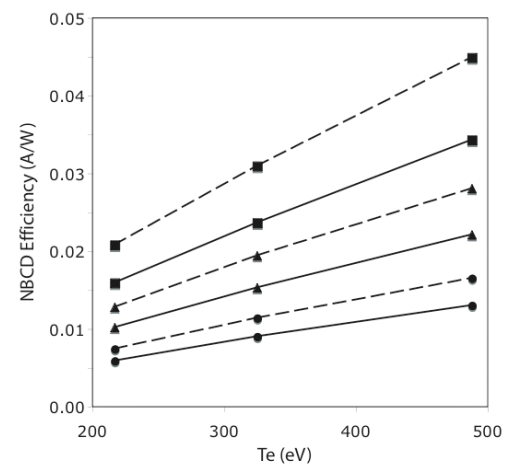

Fig. 7a. Current drive efficiency vs peak temperature (with 14590 profile held fixed) for 3 scaled densities, 14590 density profile (solid), flat density profile (dashed) 


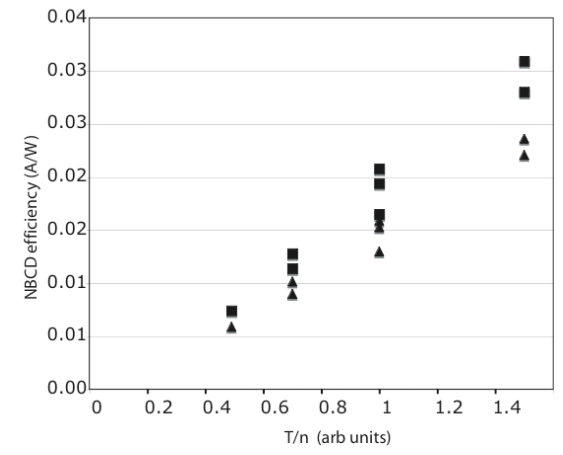

Fig. $7 b$ - Current Drive efficiency vs T/n, (Amps/watt) triangle - 14590 density profile, square - flat density profile.

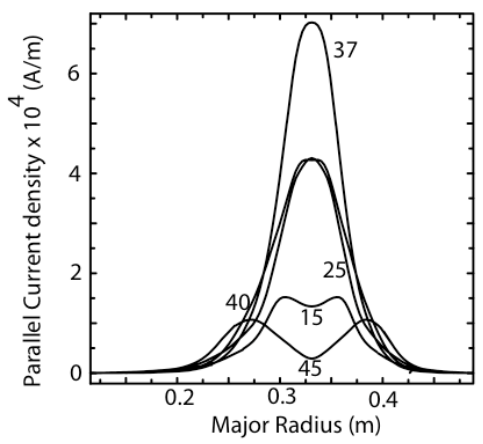

Fig. 8 Neutral beam induced parallel current density profile $(\mathrm{A} / \mathrm{m})$ for different injection angle (see definition of parallel current density). 


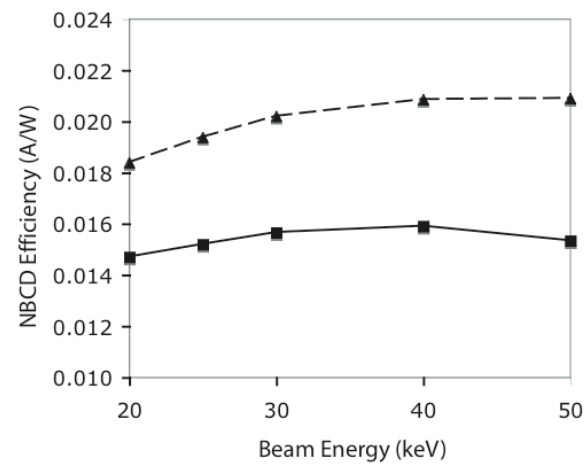

Fig. 9 Dependence of current drive efficiency on energy for 14590 density and temperature profiles (solid) and flat density and 14590 temperature profile.

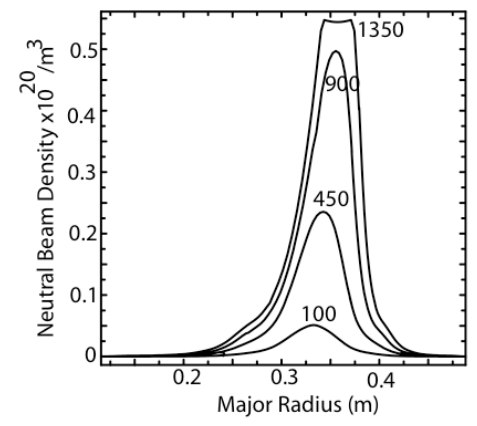

Fig. 10 Fast-ion density profile for different injected powers (kW) for 14590 profiles and 37 deg injection. 


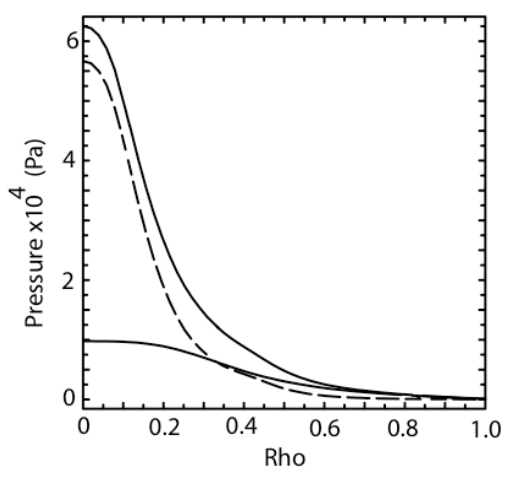

Fig. 11a Initial Plasma kinetic pressure and total pressure including fast-ion pressure vs toroidal flux coordinate, (dashed line- fast-ion pressure contribution), power injected $-450 \mathrm{~kW}$.

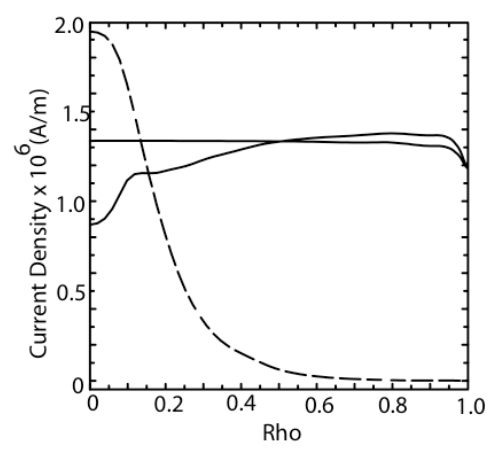

Fig. 11b Initial and final plasma parallel current density vs toroidal flux coordinate, (dashed line- NB driven current density), power injected $-450 \mathrm{~kW}$. 


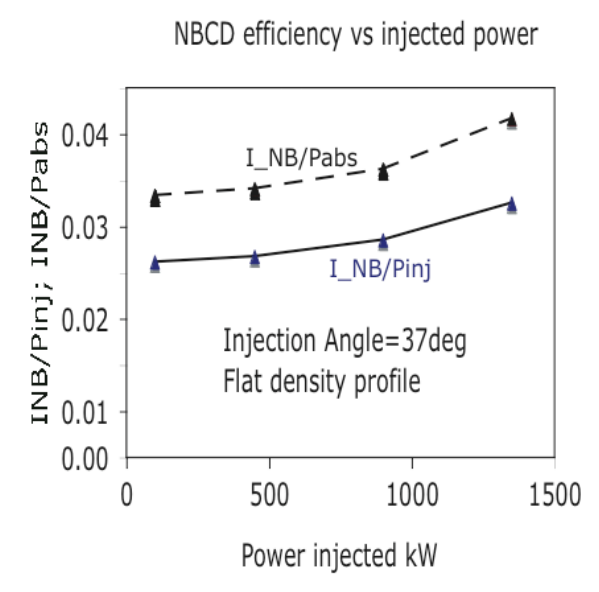

Fig. 12 - Neutral beam current drive efficiency vs power ; 14590 density and temperature profiles (solid), flat density profile and 14590 electron density profile (dashed). 37 deg injection. 


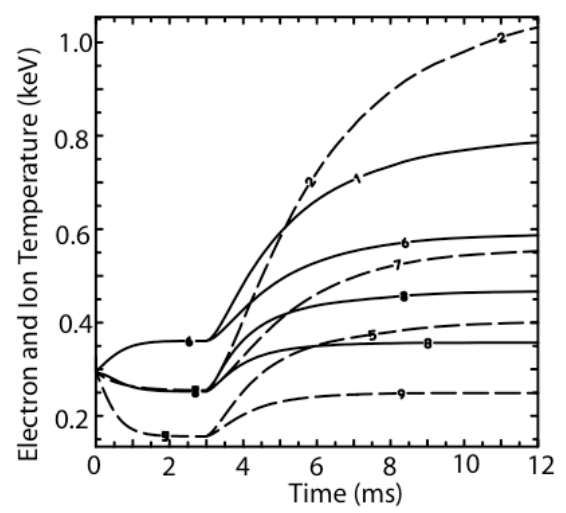

Fig. 13 Electron (solid) and Ion (dashed) temperature history. The initial time (before $3 \mathrm{~ms}$ ) is used to obtain steady state profile without beam; the beam is injected at $3 \mathrm{~ms}$. For diffusivity scales of 1.0 and 0.4 and power of 450 and $900 \mathrm{~kW}$. 


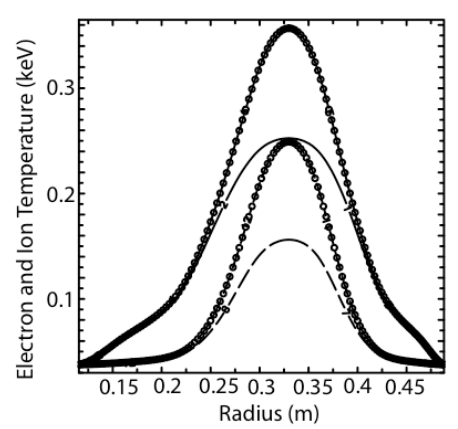

(a)

Fig. 14 Initial (line) and final (line and circles) Electron (solid) and Ion (dashed) profiles with 14590 profiles (a) $450 \mathrm{~kW}$, diffusivity scale-1.0 (b) $900 \mathrm{~kW}$, diffusivity scale- 0.4 after $9 \mathrm{~ms}$ of injection

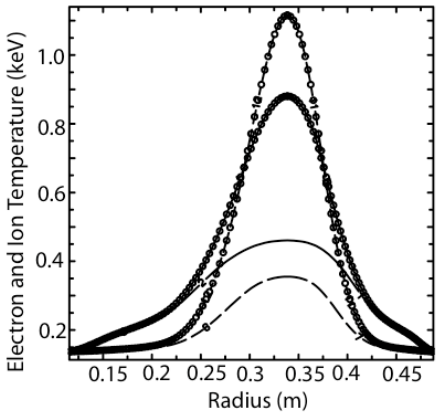

(b) 

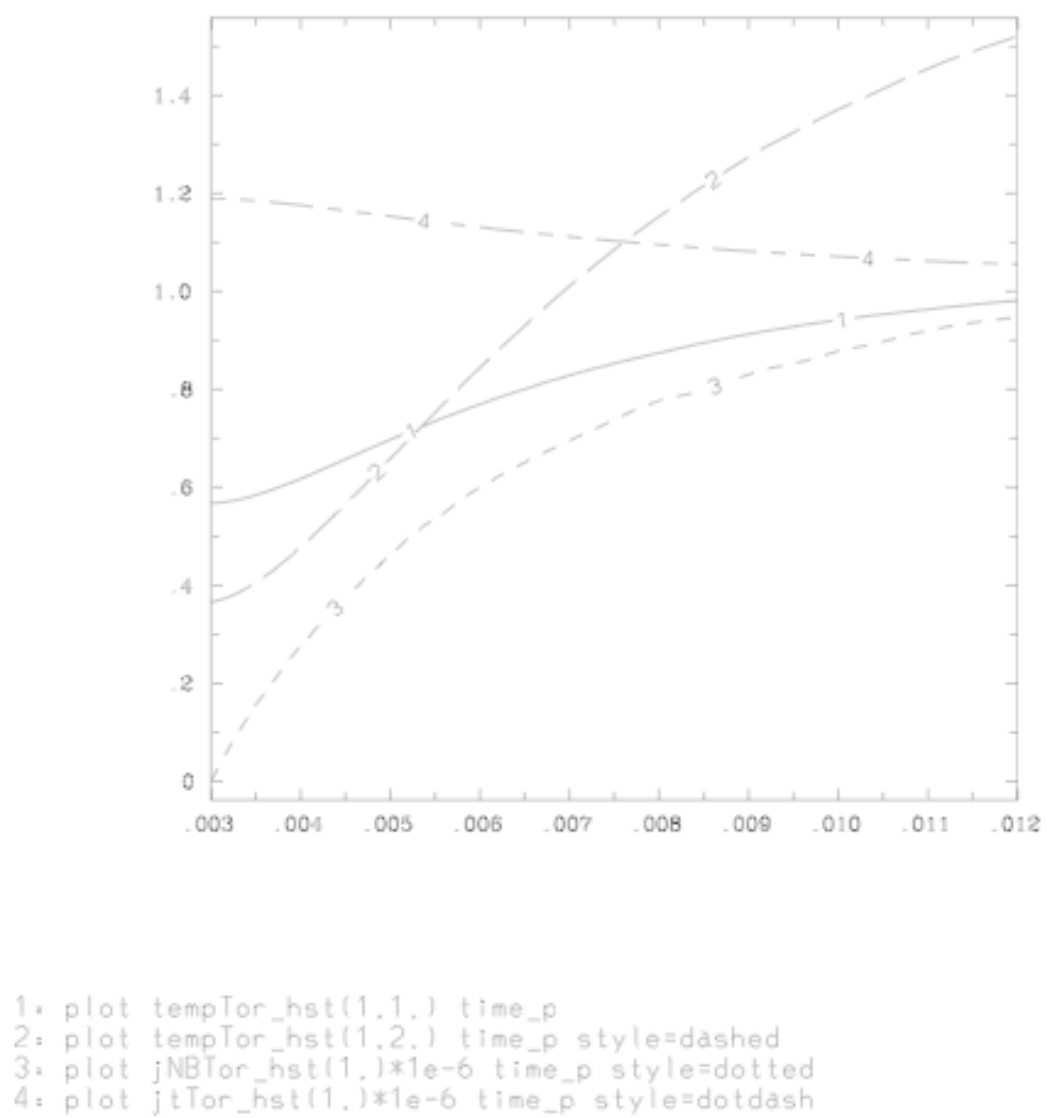

Fig. 16 - Electron (solid, curve 1) and Ion (dashed curve 2) temperatures, neutral beam driven current $(\mathrm{MA} / \mathrm{m}$ ) (dotted) vs time for $450 \mathrm{~kW}$ injected at $37 \mathrm{deg}$ into 20217 discharge target. 

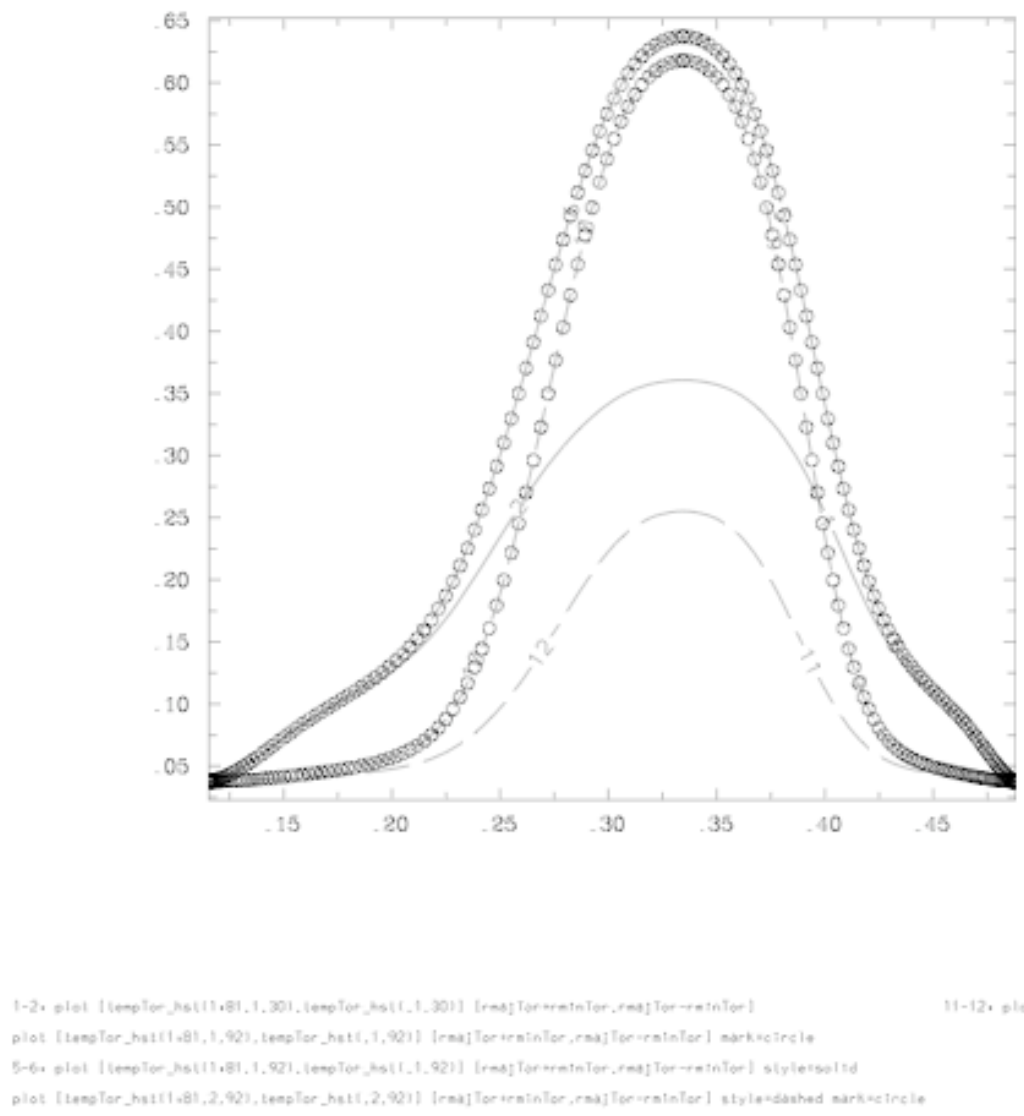

Fig. 17 - Initial (line) and final (line and circle) electron (solid) and Ion (dashed) profiles for 1.8 MW injection at 45 degree into 14590 profiles. 


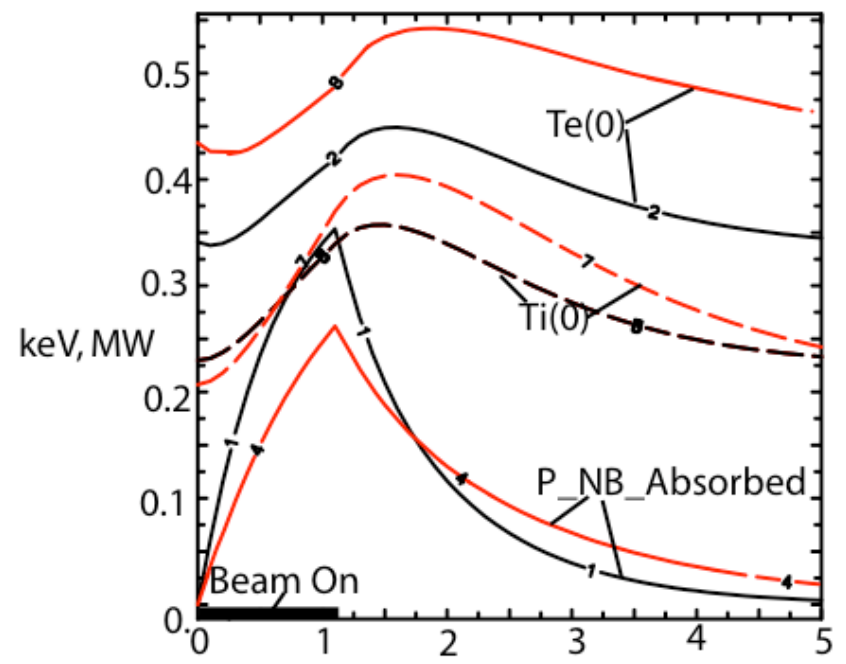

Density unscaled; Density Scaled to $70 \%$

Fig. 18 Time history for an injection of $600 \mathrm{~kW}$ for $1.2 \mathrm{~ms}$ at 37 deg angle into 14590 profiles for diffusivities adjusted to get nominal peak temperature (scaling of 0.4 ) ; black - unscaled density, red density scaled to $70 \%$. 


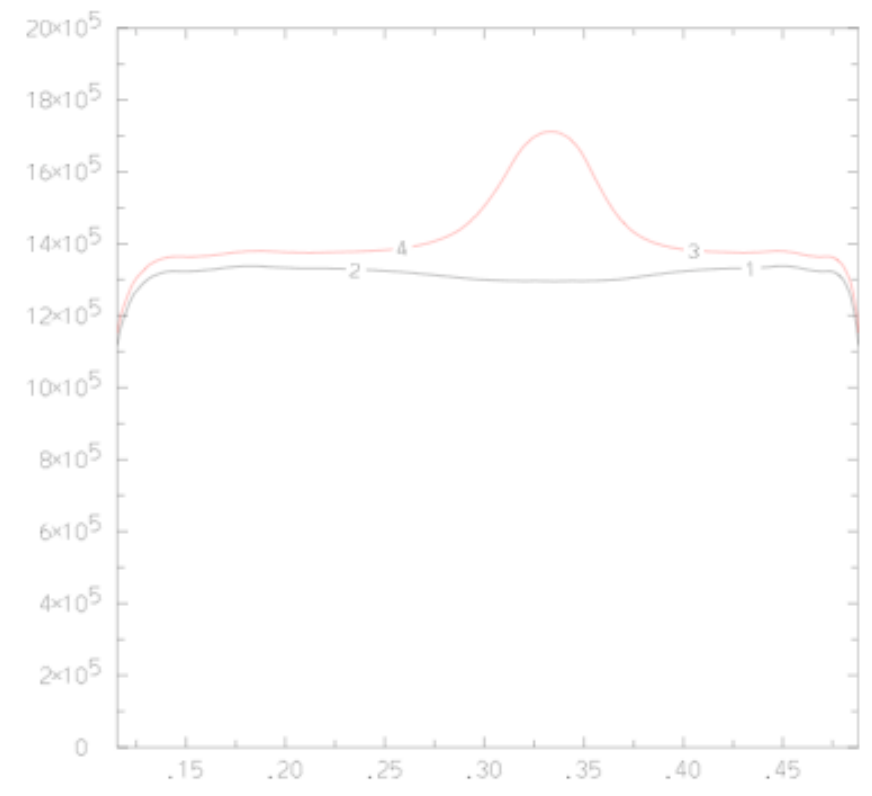

1-2= plot [jtTor_hst $(1=81,31)$.jtTor_hst (.31)] [rmajTortrminTor, rnajTor-rninTor] 3-4. plot [jtsave1.jtsave1]*100. [rseftasef, rsef-asrf]/100, colormed

eqjNB 14590

Fig. 19a Initial (black) and final reconstructed (red) equilibrium parallel current density profiles $(\mathrm{A} / \mathrm{m})$ for $450 \mathrm{~kW}$ neutral beam injection into 14590 target for 9 $\mathrm{ms}$ at $37 \mathrm{deg}$ angle. 


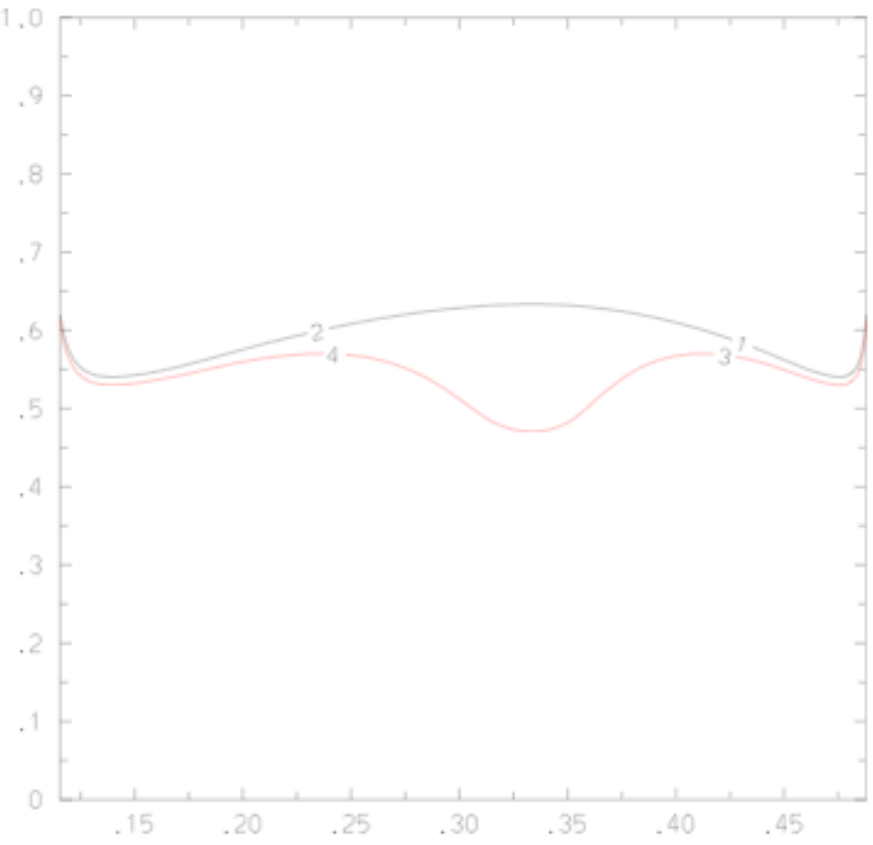

1-2. plot [oldqsave.oldqsave] [rsrftasrf, rspf-asrf]/100.

$3-4$ : plot [asave1. osave1] [rsrftasrf.rsrf-asrf]/100. color=red

eqjNB

14590

Fig. 19b Fig. 19a Initial (black) and final reconstructed equilibrium (red) safety factor profiles for $450 \mathrm{~kW}$ neutral beam injection into 14590 target for $9 \mathrm{~ms}$ at 37 deg angle. 


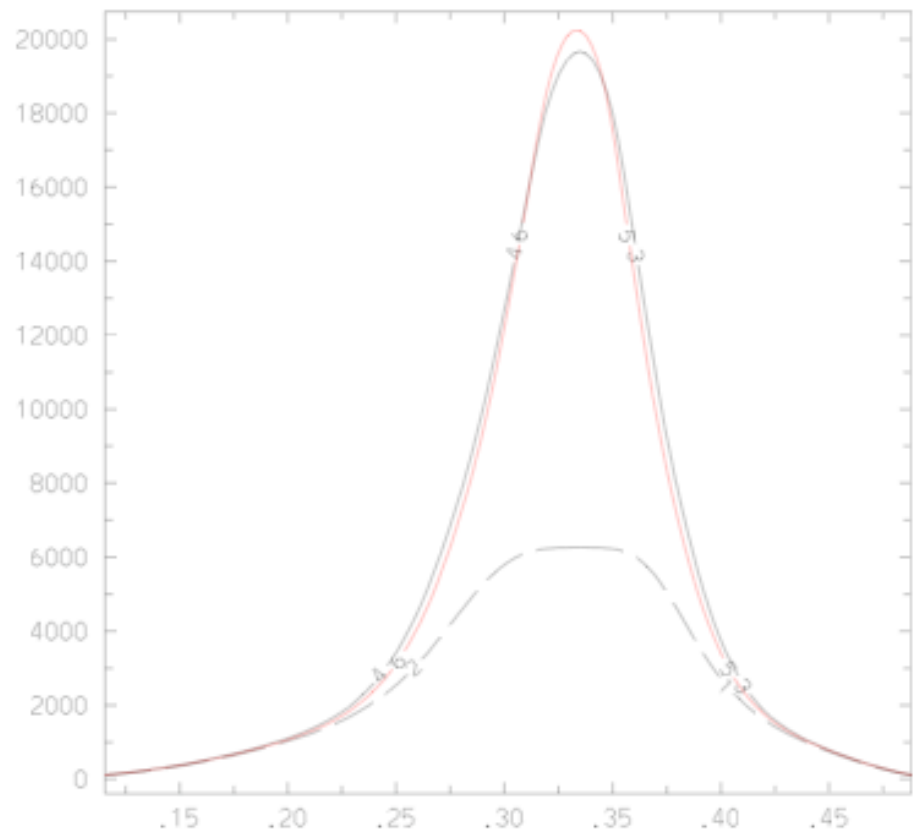

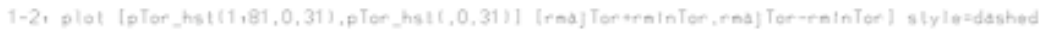

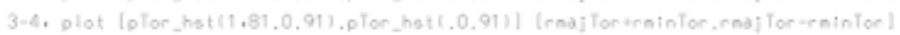

Fig. 19c- Before (black) and after reconstruction (red) equilibrium pressure profile for $450 \mathrm{~kW}$ injection into 14590 target profiles at $37 \mathrm{deg}$ for $9 \mathrm{~ms}$. The initial plasma kinetic pressure is shown (dashed). 